\title{
A LOW NOISE 2-20 GHZ FEEDBACK MMIC-AMPLIFIER
}

\author{
Herbert Zirath* ${ }^{*}$, Paulius Sakalas ${ }^{0 *}$, Jose Miguel Miranda ${ }^{+}$ \\ *Chalmers University of Technology, Department of Microelectronics, Sweden \\ "Ericsson Microwave System, Mölndal, Sweden \\ oSemiconductor Physics Institute, 2600 Vilnius, Lithuania \\ 'Dept. Applied Physics III, Faculty of Physics, University Complutense of Madrid
}

\begin{abstract}
A low noise feedback MMIC-amplifier based on a $180 \mathrm{GHz} \mathrm{f}_{\max }$ PHEMT-technology is described. The gain, input and output reflection coefficient, dc-power consumption, and noise parameters are investigated theoretically and experimentally as a function of dcbias and frequency. The noise figure is typically $2.5 \mathrm{~dB}$ with an associate gain of $22 \mathrm{~dB}$ across the $2-20 \mathrm{GHz}$ frequency range. The circuit area is less than $1 \mu \mathrm{m}^{2}$ and the dc-power consumption is lower than $100 \mathrm{~mW}$.
\end{abstract}

\section{INTRODUCTION}

For communication, radar, instrumentation, and radiometer sensors there is a need for low noise amplifiers. A versatile building block is the broad-band amplifier and at lower frequencies up to $3 \mathrm{GHz}$ there are commercially available broadband amplifiers based on both $\mathrm{Si}$ and $\mathrm{GaAs}$-technologies.

In order to achieve the lowest possible noise figure, the preferred circuit topology is the reflection noisematched input. However the bandwidth is then limited. For decade broadband amplifiers, the travelling wave concept is often used but the gain and noise performance of this type of amplifier is usually not impressive, the noise figure is of the order 6-7 dB.

The resistive parallell feedback amplifier is an interesting alternative which can yield good input and output match over a large frequency bandwidth. Such amplifier is cascadable for increased gain, and is easily made unconditionaly stable.

In this work, a resistive feedback amplifier is investigated based on a double deltadoped PHEMTprocess with $0.1 \mu \mathrm{m}$ gatelength. The process is available from a commercial foundry [1]. The aim of this work is to investigate possible gain per transistorstage, dc-power consumption, noise figure, possible obtinable bandwidth, linearity, output power etc as a function of bias. We have measured the bias-dependent noise parameters for individual transistors and extracted parameters for a noise model which can be used in commercial circuit simulators. The design was experimentally verified on MMICs fabricated at the foundry.

\section{THE RESISTIVE FEEDBACK AMPLIFIER}

The parallel resistive feedback amplifier can be analyzed by ordinary circuit analysis [2]. The relation between transconductance $g_{m}$, feedback resistance $R_{f}$ and characteristic impedance $Z_{0}$ for the condition that $\mathrm{S} 11=\mathrm{S} 22=0$ is

$$
R_{f}=g_{m} \cdot Z_{0}^{2}
$$

The gain $S_{21}$ of such an amplifier is then

$$
S_{21}=\frac{Z_{0}-R_{f}}{Z_{0}}
$$

The transistor is in this case assumed to be represented by a simple model consisting of only a current generator with transcunductance $g_{m}$. The above expression is of course a simplification which nevertheless gives a basic understanding of the underlying principle. In the simulation we use a full equivalent circuit model which also include noise sources.

\section{MMIC AMPLIFIER DESIGN}

The amplifier was designed to have a minimum gain of $20 \mathrm{~dB}$ in a frequency range of $2-20 \mathrm{GHz}$. In order to achieve this, two transistor stages were cascaded.

The gate and drain of both transistors is in this design are accessable for dc-probes. This enables an independent experimental investigation of the biastradeoff between the stages in terms of gain and noise figure. 


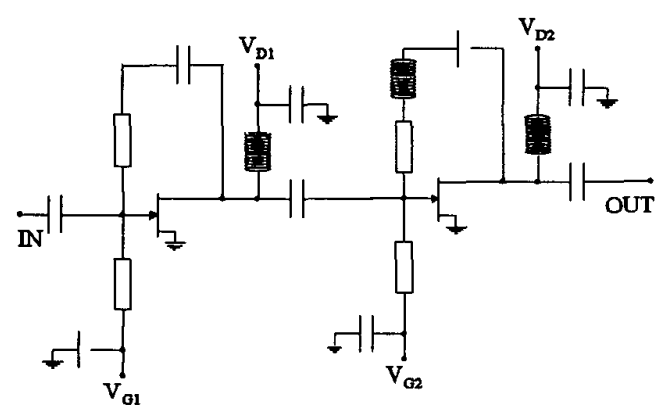

Fig.1 The circuit diagram of the feedback amplifier.

When we know the best bias points, the number of bias-connections can be minimised to only one biasconnection in order to simplify the bias access network. The circuit diagram of the feedback amplifier is shown in the fig 1. Both transistors have a gatewidth of $4 \times 50 \mu \mathrm{m}$ yielding a transconductance of nominally $160 \mathrm{mS}$. The feedback network is a resistance of 500 $\Omega$ in series with a $2 \mathrm{pF}$ capacitor. The second stage has an inductor in the feedback path in order to give the amplifier a gain boost at the high end of the frequency range. Both drains are connected to the supply voltage through an inductor of value $20 \mathrm{nH}$. The gates are biased through $250 \Omega$ gate resistors. The input and output are dc-isolated through coupling capacitances. The layout of the MMIC in shown in fig 2. input and output CPW-probes and pads for bias are provided to facilitate 'on wafer' characterization of the amplifier. The total chip area is $2 \times 1.5 \mathrm{~mm}^{2}$ while the active area of the amplifier is approximately $1 \mathrm{~mm}^{2}$.

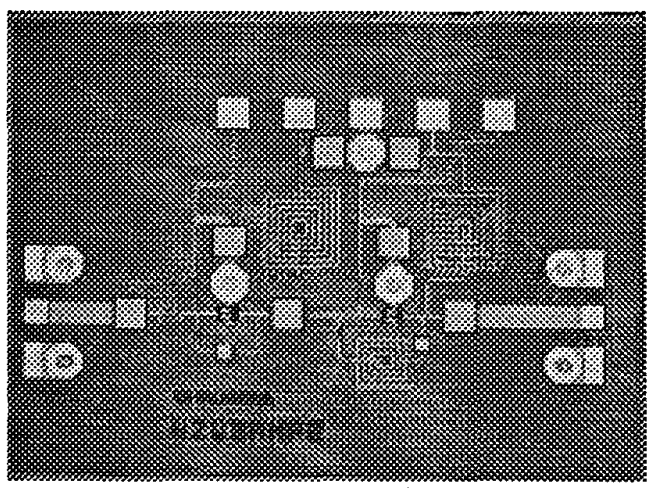

Fig. 2 Photo of the feedback amplifier.

\section{SIMULATIONS AND EXPERIMENTAI RESULTS}

The small signal parameters of the devices were obtained by means of the cold-FET method with an equivalent circuit that has been described elsewhere [3]. In addition, the noise parameters of both transistors were measured at different bias conditions. From the noise measurements, the Pospieszalski parameters, drain temperature $T_{D}$ and gate temperature $T_{G}[4]$ were initially extracted and then tuned by direct optimization with the gradient method. Once the optimized values were calculated, a non-linear curve fitting was made to obtain an analytical expression for both $T_{G}$ and $T_{D}$ as a function of the drain current density. The most accurate prediction of the noise parameters was achieved by assuming a linear dependence for $T_{G}$ and a hyperbolic dependence with a current density offset for $T_{D}$,

$$
T_{D}=T_{D 0} \cosh \frac{J_{D}-J_{0}}{J_{1}}
$$

Where $T_{D 0}, J_{0}$ and $J_{1}$ are fitting factors. The accuracy of this expression has also been verified at bias points near pinch off operation [5].

Figures 3 and 4 show the calculated drain and gate temperatures for drain voltages of 1 and 2 volts, and different drain current densities. Two transistors of different gate widths were measured to verify the scalability of the model. As it can be observed in figure 3 , the discrepancies between the drain temperatures of both devices remain low enough to safely apply the above expression with the same fitting factors to both devices.

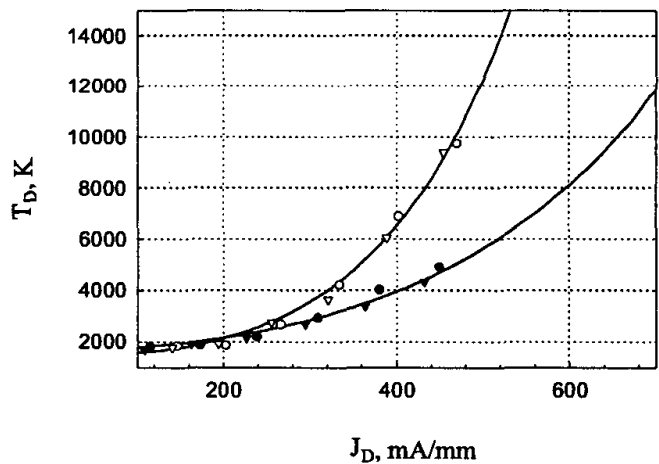

Figure 3. Optimized and fitted (lines) values of the Pospieszalski drain temperature. Circles: transistor with $100 \mu \mathrm{m}$ and triangles with $200 \mu \mathrm{m}$ gate width. Open symbols correspond to $V_{D}=2 V$, solid to $V_{D}=I V$. 
The MMIC was characterized by a vector network analyzer, HP $8510 \mathrm{C}$, and an "on wafer" noise parameter measurement system (ATN).

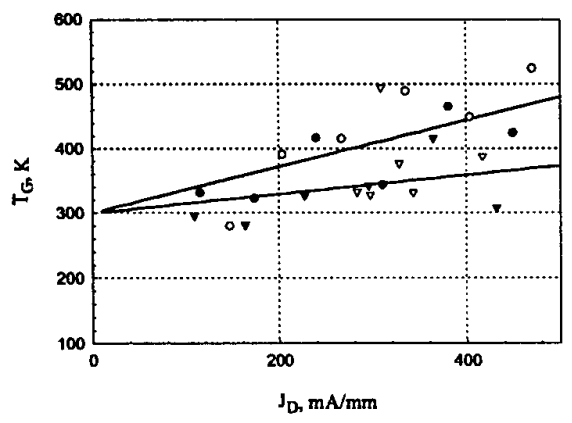

Figure 4. Optimized and fitted (lines) values of the Pospieszalski gate temperature. Circles: transistor with $100 \mu \mathrm{m}$ and triangles with $200 \mu \mathrm{m}$ gate widths. Open symbols correspond to $V_{D}=2 \mathrm{~V}$, solid to $V_{D}=1 \mathrm{~V}$.

The amplifier gain and minimum noise figure $\left(F_{\text {min }}\right)$ are both given in Fig 5 for the optimum bias conditions. The bandwidth is approximately $20 \mathrm{GHz}$ and the noise figure was about $2.6 \mathrm{~dB}$. Lines represent the simulation made by exploiting a commercially available CAD tool [6]. These simulations could accurately predict both the measured minimum noise figure and gain, and this fact motivated us to investigate the performance that should be expected for a similar amplifier, but designed with a larger first stage transistor (6x50 micron gate width) and a higher feedback resistor $(1500 \mathrm{Ohm})$.

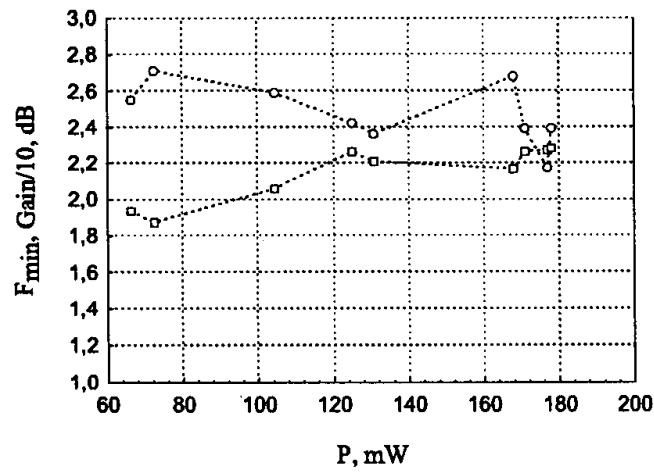

Fig.5 Measured (open symbols) and simulated (ines) minimum noise figure and gain of the wideband amplifier versus frequency. Drain bias of the first transistor $V_{D 1}=1.5 \mathrm{~V}$, for the second $V_{D 2}=1.5 \mathrm{~V}$, gate biases $V_{G I}=O V, V_{G 2}=0 V$.
These modifications led to an improved noise figure, $0.2 \mathrm{~dB}$ less than that obtained in the first design, and also to an slightly higher gain, especially at the lower frequencies.

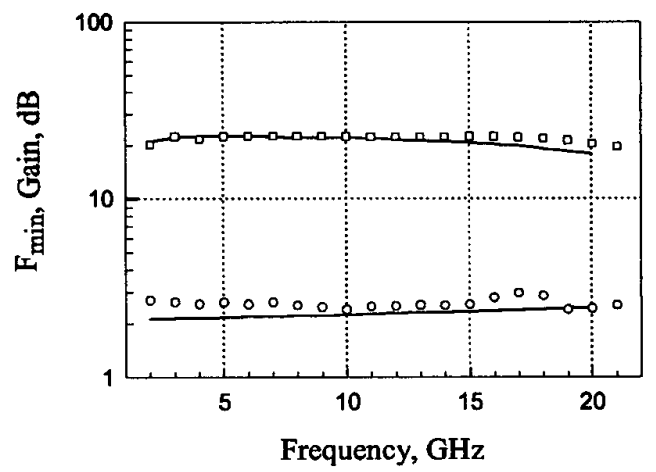

Fig. 6 Measured noise and gain of the amplifier versus power consumption. Open circles stands for $F_{\text {min }}$ open squares - gain/10, dotted line - guide for an eye. The values are taken at $10 \mathrm{GHz}$ frequency.

The gain and $F_{\min }$ of the fabricated amplifier showed a fairly slight bias dependence. This can be observed in Fig. 6, where the measured gain and noise are both plotted as a function of the dc-power. The best performance of the amplifier is obtained at the $125 \mathrm{~mW}$ of consumed power, the performance is however acceptable at a power as low as $70 \mathrm{~mW}$. The optimal drain bias was found to be $1.5 \mathrm{~V}$ for both transistors.

\section{SUMMARY}

A MMIC broadbandamplifier based on a $180 \mathrm{GHz} \mathrm{f}_{\max }$ P-HEMT-technology was designed, fabricated and characterized. The amplifier has a gain of more than 20 $\mathrm{dB}$, an input and output reflection coefficient less than $10 \mathrm{~dB}$, a bandwidth of more than $20 \mathrm{GHz}$ and a noise figure of less than $2.7 \mathrm{~dB}$ with a dc-power consumption around $100 \mathrm{~mW}$. The simulation of the amplifier noise figure was made by using the Pospieszalski model with unified bias dependent expressions for the drain and gate temperatures. This approach enabled an accurate prediction of the overall amplifier noise performance.

\section{ACKNOWLEDGEMENTS}

The Swedish Foundation for Strategic research, SSF, and Chalmers Center for high speed Electronics, $\mathrm{CHACH}$, is acknowledged for the funding of this project. 


\section{REFERENCES}

[1] Process D01PH from Philips Micronde Limeil.

[2] G. Gonzales, Microwave amplifiers, Prentice Hall.

[3] M. Garcia, N. Rorsman, K. Yhland, H. Zirath, I. Angelov, "Fast, Automatic and Accurate HFET Small-Signal Characterization", Microwave Journal, July 1997, pp. 102-117.

[4] M. W. Pospieszalski, "Modeling of Noise Paramaters of MESFETs and MODFET and their Frequency and Temperature Dependence", IEEE Trans. on MTT, vol 37 no 9,1989 , pp. 1340-1350.

[5] J. M. Miranda, H. Zirath, J. L. Sebastián, "Noise modelling of $0.15 \mu \mathrm{m}$ gate length HFETs based on InGaAs channels", submitted to the GHz 2000 Symposium, March 2000, Goteborg, Sweden.

[6] MNS, Hewlett-Packard Microwave Nonlinear Simulator (7-th release), HP Part No.8515090220, manual 4-th edition, printed in U.S.A., HP EEsof Division, 1400 Foutaingrove Parkway, Santa Rosa, CA 95403, U.S.A., 1996). 